\title{
Power-law singularity as a possible catastrophe warning observed in rock experiments
}

\author{
Sheng-Wang Hao ${ }^{\text {a,d }}$, Feng Rong ${ }^{a}$, Ming-Fu Lu ${ }^{a}$, Hai-Ying Wang ${ }^{a}$, Meng-Fen Xia ${ }^{\text {a,b }}$, \\ Fu-Jiu Ke ${ }^{\mathrm{a}, \mathrm{c}}$, Yi-Long Bai ${ }^{\mathrm{a}, *}$ \\ a State Key Laboratory of Nonlinear Mechanics (LNM), Institute of Mechanics, Chinese Academy of Sciences, Beijing 100190, China \\ ${ }^{\mathrm{b}}$ Department of Physics, Peking University, Beijing 100871, China

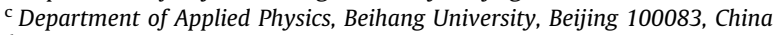 \\ d School of Civil Engineering and Mechanics, Yanshan University, Qinhuangdao 066004, China
}

\section{A R T I C L E I N F O}

\section{Article history:}

Received 30 August 2011

Received in revised form

23 May 2012

Accepted 17 December 2012

Available online 17 February 2013

Keywords:

Power law singularity

Catastrophic rupture

Precursor

Specific warning

Earthquake forecasting

\begin{abstract}
A B S T R A C T
The question which type of signals can be determinately related to catastrophic rupture in heterogeneous brittle media still remains open. Here we report a specific precursor of catastrophic rupture, i.e. a power-law singularity of responses, based on rock experiments. Our experimental observations show that the singularity with power exponent $-\beta$, where $\beta=0.51 \pm 0.10$ (mean \pm s.d.), appears ahead of catastrophic rupture in some rocks, and the singularity does not appear at all for gradual failure. It is indicated that the power-law singularity can emerge well only close to catastrophic rupture and thus it could serve as a specific warning for catastrophic rupture. To address the potential forewarning of imminent catastrophic rupture, a fitting process based on the data before catastrophic rupture was developed to determine the two unknowns related to the occurrence, catastrophe point $U_{F}$ and the power exponent $-\beta_{u}$. It is demonstrated that the power-law singularity appears only in the vicinity of catastrophe, and that the power-law singularity does not occur for gradual failure.
\end{abstract}

(c) 2012 Elsevier Ltd. All rights reserved.

\section{Introduction}

Catastrophic events in geological media, such as the recent big earthquakes in China (M8.0 2008), Chile (M8.8 2010) and Japan (M9 2011), are a major concern of global public. Unfortunately, no warnings were made before these big catastrophic events, since the knowledge on reliable precursors prior to the catastrophic rupture is still lacking [1-9]. It is well-known that in both Nature and Science there have been special debates on whether or not earthquakes can be predicted [1-3] and a NAS Colloquium entitled "Earthquake prediction: The Scientific Challenge" has been organized [4]. Additionally, as Main [3] asked, "How far should we go in investigating the degree of predictability that might exist"?

Since then, to identify which type of signals could be uniquely related to the occurrence of catastrophic rupture in geological media has become a key to the problem. It is demonstrated that the micro-events evolution could exhibit some scaling laws [2] and the limiting distribution of the size of each episode (distribution of the number of contributing cracklets) has an asymptotic power-law distribution [10]. Also, a continuum

\footnotetext{
* Corresponding author at: State Key Laboratory of Nonlinear Mechanics (LNM), Institute of Mechanics, Chinese Academy of Sciences, Beijing 100190, China. Tel: +86 10 82543939; fax: +861082543977.

E-mail address: baiyl@lnm.imech.ac.cn (Y.-L. Bai).
}

damage mechanics model was introduced to explain temporal scaling laws in brittle fracture and seismicity and revealed the key role of occurrence and coalescence of microcracks [11]. It is also stressed [11-13] that the critical behavior depends on microscopic features, such as the geometry and type of the bonds.

A number of options, such as accelerated responses [6-8, 12,14-22], spinodal-like instability [23] and a first-order [24] or second-order [25] phase transition, have been proposed to understand the rupture in crust. In particular, a number of researchers have found that the rupture is usually preceded by a power-law time-to-failure in the cumulative Benioff strain, $\left(t_{F}-t\right)^{m}$, where $t_{F}$ is the time of failure. In particular, Sornette [26] found the mean field value to be $m=1 / 2$ in a critical phase transition, but Rundle et al. [23] demonstrated a value of $m=1 / 4$ in a spinodal transition. On the other hand, observational studies [8] indicated that $m \approx 0.3$ for large earthquakes. On the other hand, some recent results $[27,28]$ argued that whether or not Accelerating Moment Release is a statistically significant precursor to large earthquakes, and the existence of Accelerating Moment Release is still debated [28]. Thus, the key issue is what sort of accelerated criticality can specifically identify the occurrence of catastrophic rupture [29-32].

To understand the mechanism of catastrophic rupture like earthquake and investigate the way to forecast the catastrophe, numerous rock experiments were conducted by many researchers 
in laboratory [33-37]. It was claimed that these model earthquakes should provide a better understanding of the mechanisms that may lead to large seismic events in the real world [35-36]. Of course, there are some important differences between the fracture of rock in compression experiments and an earthquake rupture. It was stated [38] that the straining mechanism in the laboratory for crystalline rocks occurs by local microcracking, and natural seismicity occurs by shear on pre-existing faults, so the quantitative behaviour may be significantly different in detail. However, there are many similarities between the compression test in lab and the compressional ones in the Earth. It has long been recognized that acoustic emissions due to microcracking in the laboratory could provide a useful analogue for the examination of earthquake sequences [38-40]. Furthermore, the stored elastic energy might drive the unstable catastrophic rupture in rock test in lab and Earth's crust.

In this paper, we report a possible precursor of catastrophic rupture, i.e. a power-law singularity of responses, based on rock experiments. Our experimental observations show that a powerlaw singularity, $R_{u}=d u / d U \propto\left(1-U / U_{F}\right)^{-\beta_{u}}$, where $\beta_{u}=0.51 \pm 0.10$ (mean \pm s.d.) ( $-\beta_{u}$ will be called the critical exponent), appears ahead of catastrophic rupture and that the power-law singularity does not occur for gradual failure.

\section{Description of experiments}

In our experiments, two types of rock samples (granite and marble) with sizes of $16 \times 20 \times 40 \mathrm{~mm}^{3}$ were compressed uniaxially in the $40-\mathrm{mm}$ direction by using an Instron 1195 testing machine. The loading mode was displacement control with a resolution of $0.15 \mu \mathrm{m}$, and the crosshead speed was $0.05 \mathrm{~mm} / \mathrm{min}$. The displacement of the crosshead is the governing displacement $U$ that combines the deformation of the loading apparatus and the deformed rock sample. Catastrophic rupture occurs at a critical displacement $U_{F}$. The deformation $u$ of the rock sample was measured by an extensometer with a resolution of $1 \mu \mathrm{m}$ and an offset load of $1 \mathrm{kN}$.

In our experiments, the deformation $u_{F}$ of the sample at the catastrophic rupture point was approximately $0.2 \mathrm{~mm}$, but the total combined deformation $U_{F}$ of both the sample and testing apparatus at the catastrophic rupture point was about $2 \mathrm{~mm}$ (A typical force-displacement curve of a rock test is shown in Fig. 1) because the stiffness of the testing apparatus was much lower than that of the sample.

Moreover, because the rupture of all rock specimens in our experiments appeared to be catastrophic rather than gradual, we also performed some tests on concrete samples showing gradual

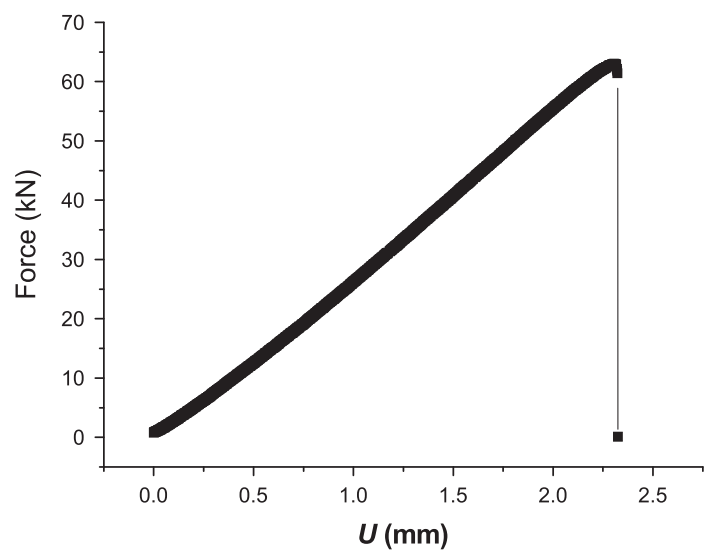

Fig. 1. A typical force-displacement curve of a rock test. rupture as a comparison to validate the finding that the powerlaw singularity appears specifically ahead of catastrophic rupture.

Each concrete specimen was a rectangular block, $160 \mathrm{~mm}$ high and $60 \mathrm{~mm} \times 60 \mathrm{~mm}$ in cross-section. Each specimen was cast in an accurately machined steel mold. The components of the concrete were silicate cement (28-day strength of $42.5 \mathrm{MPa}$ ), fly ash, natural river sand and limestone coarse aggregate $(5-10 \mathrm{~mm}$ in size). The mixture proportion by weight was as follows: cement:water:fly ash:fine aggregate:coarse aggregate $=1: 0.71$ : 0.43:2.71:3.71. The specimens were cured for 6 days in a fog room at $20 \pm 2 \mathrm{C}$ and relative humidity $(\mathrm{RH}) \geq 95 \%$. Then, the specimens were uniaxially compressed in the vertical direction $(160 \mathrm{~mm})$ with a uniformly increasing displacement.

\section{Characteristics of responses}

There are many dependent variables, including deformation, cumulative energy release, and damage, when a sample is loaded with a controlling displacement $U$ or a force. In our experiments, we took the displacement $U$ as the governing variable. Obviously, the response that can be most easily and directly measured in experiments is the deformation $u$ of the sample. Also, $R_{u}$ may be a good parameter to use as a measure of incremental slip. For example it does not have the disadvantage of having to analyse cumulative (and hence correlated) data such as the cumulative slip, which can produce biased results [41]. In this paper, we used the response function $R_{u}=\mathrm{d} u / \mathrm{d} U$ of the sample deformation $u$ to examine the power-law singularity ahead of catastrophic rupture. If the testing apparatus was infinitely stiff, the deformation $u$ of the sample would be equal to the boundary displacement $U$, and its response $R_{u}$ would remain constant. As shown in some theories in rock mechanics, there would be no catastrophic rupture under absolutely stiff loading. In fact, under certain conditions, catastrophic rupture may be triggered by damage localization even in an absolutely stiff testing machine [42-45]. However, it should be noted that the testing apparatus used in the experiments is elastic (to simulate most practical cases) with a stiffness of about $60 \mathrm{kN} / \mathrm{mm}$, which is about $1 / 10$ of the intact sample stiffness; thus, catastrophic rupture appears under certain conditions.

The curves of normalized force $P / P_{\max }$ and response $R_{u}=\mathrm{d} u / \mathrm{d} U$ versus the normalized governing displacement $U / U_{F}$ are shown in Fig. $2 \mathrm{a}$ and $\mathrm{b}$ shows the evolution curve $u / u_{F}$ versus $U / U_{F}$ of marble 1 as an example, where the subscripts max and $F$ denote the maximum load and rupture, respectively. It is clear that all samples showed a sharp increase in the response $R_{u}=\mathrm{d} u / \mathrm{d} U$ before catastrophic rupture as shown in Fig. 2c.

Now, let us have a close examination of the variations of the load and sample deformation with increasing displacement $U$ by means of Figs. 2a and 3. It can be seen that, at a very early stage of the compression test, the force-displacement curve is slightly convex upward (see Fig. 2a; also see $[46,47]$ ). In this portion up to about $30 \% U_{F}$, the deformation-displacement (i.e., $u-U$ curve) is slightly concave downward (see Fig. 2b), and the responses $R_{u}=\mathrm{d} u / \mathrm{d} U$ decrease monotonically in the log-log plots of $R_{u}-\left(1-U / U_{\mathrm{F}}\right)$. This stage is related to the elastic closing of cracks [47]. Later, an almost linear force-displacement relation and a nearly linear $u-U$ relation follows (see Fig. $2 \mathrm{~b}$ ), and the response $R_{u}=d u / d U$ correspondingly evolves slowly (see Fig. 2c). Thus, the slope remains at a low value (see the insets of Fig. 3 ). Fig. 3 also shows the log-log plots of the response $R_{u}=\mathrm{d} u / \mathrm{d} U$ versus the reduced displacement $\left(1-U / U_{\mathrm{F}}\right)$ of five specimens. This region ranges from $30 \% U_{F}$ to $70 \% U_{F}$ (see the insets in Fig. 3). Then, in the third region, the slope of the force-displacement curve decreases, and later, the deformation $u$ of the sample 
a
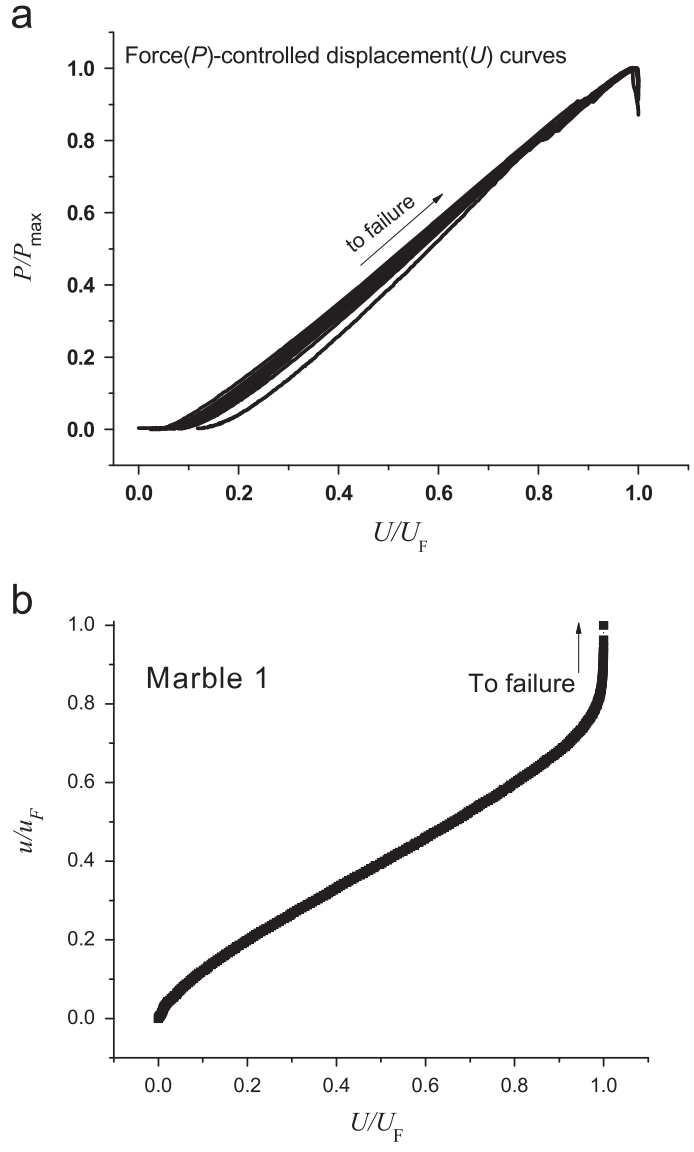

C

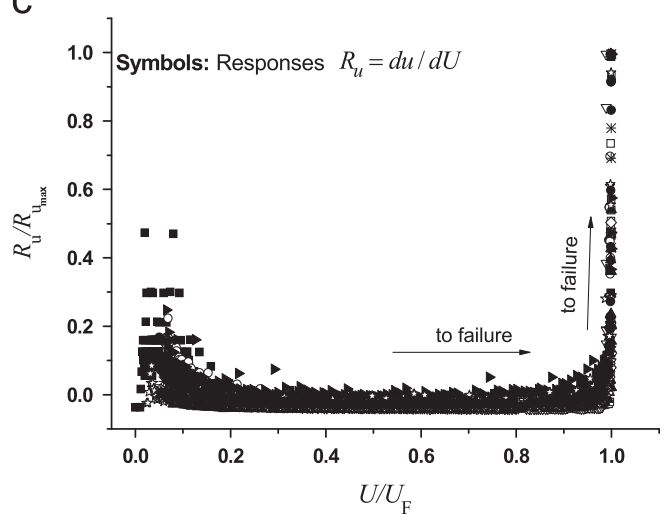

Fig. 2. Responses observed in experiments. (a) The normalized force $\left(P / P_{\max }\right)$ displacement $\left(U / U_{F}\right)$ curves for ten rock samples. (b) The normalized deformation $\left(u / u_{F}\right)$-controlling displacement $\left(U / U_{F}\right)$ curves for Marble 1 . (c) The behavior of responses $R_{u}=\mathrm{d} u / \mathrm{d} U$ versus controlling displacement $\left(U / U_{F}\right)$ for the ten rock samples shown in Fig. 2(a).

increases rapidly until catastrophic rupture. This region is comprised of two portions: (I) the slope of the deformationdisplacement curve (i.e., the $u-U$ curve) increases progressively from a low value with increasing boundary displacement $U$; and (II) the slope of the deformation-displacement curve (i.e., the $u-U$ curve) increases rapidly (Fig. 2b) and exhibits nearly linear behavior in the log-log graph of $R_{u} \sim\left(1-U / U_{\mathrm{F}}\right)$ as shown in Fig. 3, namely, a power-law singularity. This should be attributed to the accumulation and coalescence of microcracks and microdefects, as pointed out in previous studies [10,40].

One important issue at rupture is that no accumulated quantity can physically tend to infinity at rupture $[11,15,38]$. Thus, any critical behavior leading to the divergence of accumulation at rupture would not be valid. In particular, to fully justify what kind of critical behavior that will definitely appear ahead of catastrophic rupture becomes an urgent task. To do so, in the following sections, we will more closely examine the evolution of response $R_{u}$ experimentally.

First, let us focus on how fast the response $R_{u}=\mathrm{d} u / \mathrm{d} U$ grows with the controlling displacement $U$ ahead of catastrophic rupture. From the linear dependence in the left part of the log-log plots in Fig. 3, we can see that the increase in the responses ahead of catastrophic rupture can be described by a power-law singularity,

$R_{u}=\mathrm{d} u / \mathrm{d} U \propto\left(1-U / U_{\mathrm{F}}\right)^{-\beta_{u}}$.

In particular, it can be seen from Figs. 2 and 3 that the slope decreases from almost zero at about $70 \% U_{F}$, and the power exponent $-\beta_{u}$ becomes about -0.5 near catastrophic rupture. To observe how the slope decreases, the evolving response is zoomed into the last $5 \%$ of $U / U_{F}$, as shown in Fig. 4. It should be noted that the linear fitting in Fig. 3 was made step by step, and the window size in every fitting varies with increasing $U$, i.e., every fitting starts from the same point $\left(70 \% U_{F}\right)$ but ends at the individual observation point. Fig. 5 shows the fitting results for five specimens, and the average values of $\beta_{u}$ are presented in Fig. 6. It is obvious that the power-law exponent $\beta_{u}$ vary from about zero to almost 0.5 , and the fitting error also decreases progressively when the deformation evolves to catastrophic rupture. In particular, the fitting error is very large when the observed points are far from catastrophic rupture. Thus, the fitting results are dominated by the rapid increase near catastrophic rupture. It should be mentioned that in a true prospective scenario neither of $\beta_{u}$ and $U_{F}$ is known, and this will significantly degrades the forecasting power. Bell et al. [48] have looked at this problem, discussed the appropriate techniques to use in fitting data, and showed that predictability was very much degraded in true prospective mode when the failure time is not known. In Section 4, five rock samples were used to demonstrate this process.

Clearly, the fitting of the critical power-law exponent is valid only close to catastrophic rupture. Based on the experimental data (Figs. 2 and 3), we found that a best-fitting of the critical exponent $-\beta_{u}$ can be obtained within the last $4 \%$ of the entire deformation range ahead of the catastrophic point $U_{F}$. An unweighted least squares linear regression was performed to find the exponents. The graphs of the best-fit $\beta_{u}$ calculated by a moving window (for a fixed window with a size of about $4 \%$ of the entire deformation range) and the corresponding standard error are presented in Fig. 7. It can be seen that the linear fitting errors are very large when the sample deformation is far from catastrophic rupture, but the errors decrease rapidly near catastrophic rupture. Thus, the linear fitting is effective just ahead of catastrophic rupture, i.e., the power-law singularity can only present well close to catastrophic rupture.

Then, we fitted all experimental data for forty-three marble and granite samples to the power law in the range of about three orders of magnitude from $U / U_{F}=0.96$ to rupture (i.e., $-4<$ $\left.\log _{10}\left(1-U / U_{\mathrm{F}}\right)<-1.4\right)$ and obtained the mean critical exponent $\beta_{u}=0.51$ with standard error \pm 0.10 by taking an unweighted mean. The mean value is defined as $\left\langle\beta_{u}\right\rangle=$ $(1 / 43)\left[\Sigma_{i}^{23}\left(\beta_{u}\right)_{i}+\Sigma_{i}^{20}\left(\beta_{u}\right)_{j}\right]$, and the standard error is calculated by $\sqrt{(1 / 43)\left[\Sigma_{i}^{23}\left(\left(\beta_{u}\right)_{i}-<\beta_{u}>\right)^{2}+\Sigma_{i}^{20}\left(\left(\beta_{u}\right)_{j}-<\beta_{u}>\right)^{2}\right]}$, where $i$ and $j$ are the number of granite and marble specimens, respectively. The fluctuations, which were also observed in the experiments reported by Guarino et al. [13,49], are partly due to the strong oscillations of the control quantity near brittle rupture. The statistics for the fitting of all 43 samples are shown in Table 1. It is clear that 
a

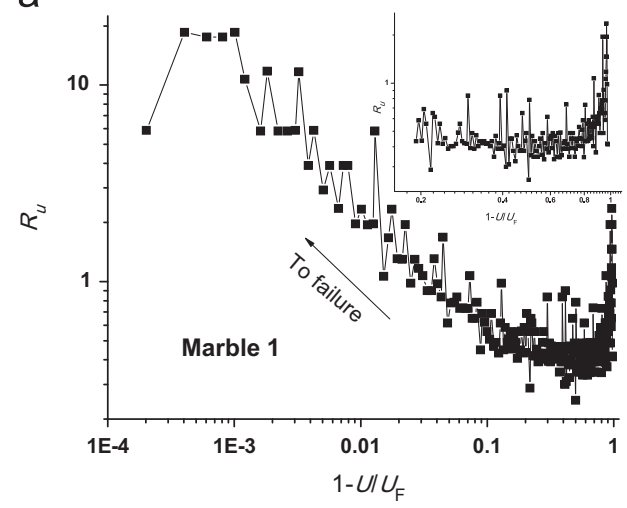

C

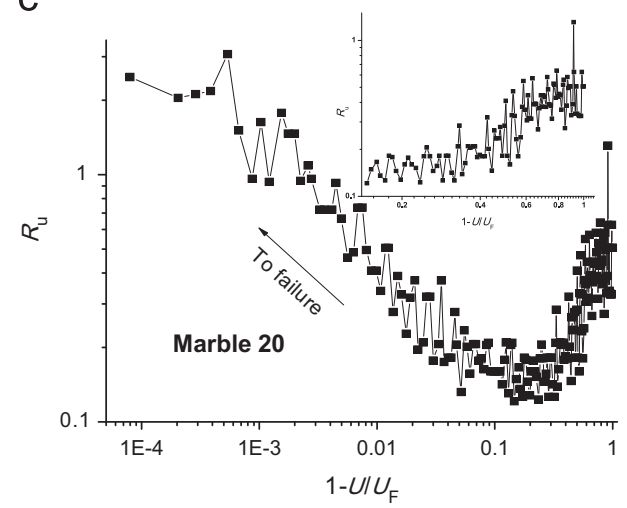

b

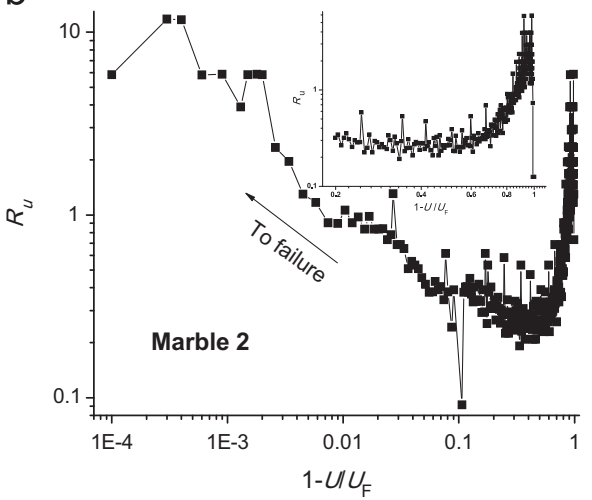

d

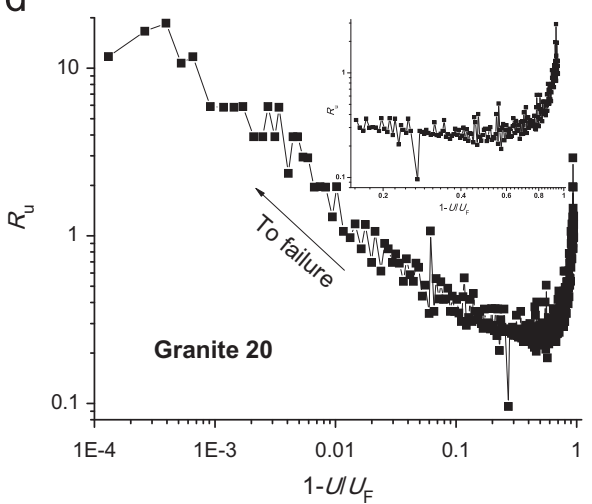

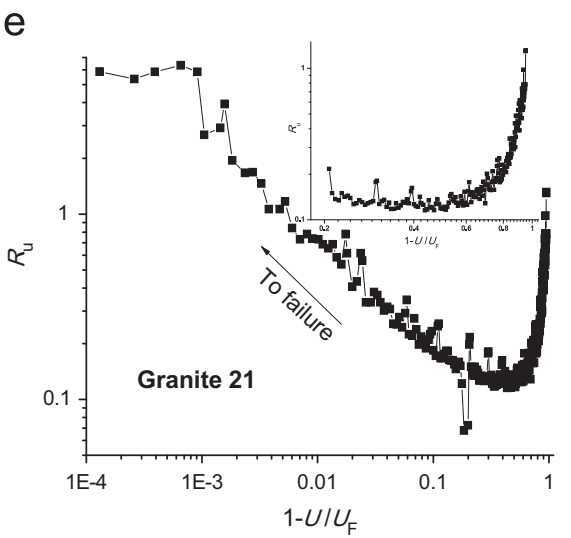

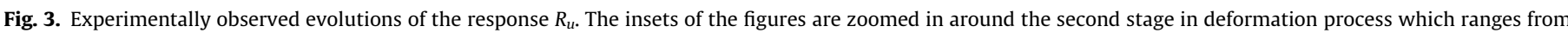
$30 \% U_{F}$ to $70 \% U_{\mathrm{F}}$.

our experimental results show a power-law singularity that always appears in advance of catastrophic rupture, with some variations. It should be noted that the range of $0.96 U_{F} \leq U \leq U_{F}$ corresponds to a time interval depending on a loading rate that could be long enough for practically important cases under engineering conditions when the interval of the accelerating nucleation is long enough. To illustrate the fitting in detail, the experimental data and their fittings for three samples are shown in Fig. 8.

On the other hand, Fig. 9 shows the experiment for concrete which resulted in with no catastrophic rupture. Although there is a part of the curve exhibiting a rapidly increasing response (as shown in Fig. 9b), it should be noted that the increasing rate of $R_{u}$ will decrease afterward, and the slope of the log-log curve of $R_{u} \sim 1-U / U_{\left(R_{u}\right)_{\max }}$ will tend to zero at the maximum response point, as shown in Fig. 9a and b. To further clarify the evolution behavior of exponent, the fitting of the slopes of the $\log \left(R_{u}\right)$ versus $\log \left(1-U / U_{R_{\max }}\right)$ curve and their standard errors for concrete were performed with a moving window of fixed size $4 \% U_{R_{\max }}$ (as shown in Fig. 9c). It is obvious that the response functions do not present any critical power-law singularity ahead of the maximum response point, but they tend to zero near the maximum response point.

\section{Short-term forewarning of catastrophic rupture}

To address the potential forewarning of imminent catastrophic rupture, a fitting process for the data before catastrophic rupture was developed to look for the unknown parameters $A, U_{F}$ and $\beta_{u}$ yielding the best-fitting $R_{u}=A\left(U_{F}-U\right)^{-\beta_{u}}$. Five rock samples were used to demonstrate this process. In every fitting, the data ranged from $96 \%$ of $U_{a}$ to $U_{a}$, where $U_{a}$ is the boundary displacement, 
beyond which the data were assumed not to be available. To justify the forecast possibility of the fitting process, a normalized fitting function $R_{u}=A^{P}\left(U_{F}^{P}-U / U_{F}\right)^{-\beta_{u}}$ was used, where $U_{F}^{P}$ is the boundary displacement at the predicted catastrophe point normalized by the true value of the catastrophe point $U_{F}$.

The Levenberg-Marquardt algorithm (LMA) was adopted to find the optimal values of the parameters $A^{P}, U_{F}^{P}$ and $\beta_{u}$, and the initial values for the parameters were assigned as $A^{P}=1.0, U_{F}^{P}=U_{a} / U_{F}$ and $\beta_{u}=0.5$. Two fitting results for granite-21 are shown in Fig. 10. It can be seen that when $U_{a} / U_{F}=0.99$ rather than 1 , the fitted results are still not good because the error in $\beta_{u}$ remains very large (i.e., the power law does not work) as shown in Fig. 10a. However, when

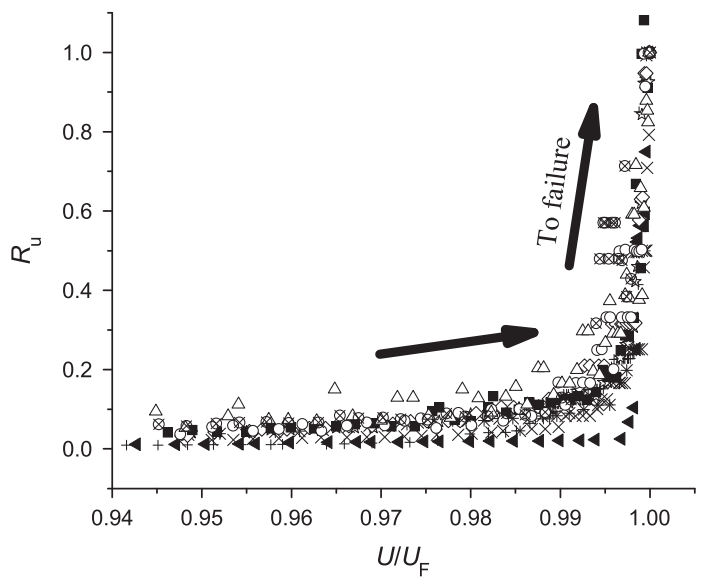

Fig. 4. Evolution of the response $R_{u}$ zoomed in to the last $5 \%$ of $U / U_{F}$ ahead of catastrophic failure (five samples).
$U_{a} / U_{F}=1$, the fitting error of $\beta_{u}$ becomes much smaller, i.e., the power law presents well, and the predicted value of $U_{F}^{P}$ is close to 1 , as shown in Fig. 10b. Such results imply that the power-law singularity appears only in the vicinity of catastrophe, and a shortterm forewarning of catastrophe might be possible.

To further validate the forewarning of catastrophic rupture in terms of the present method, the fitted results for five samples corresponding to different $U_{a}$ are shown in Fig. 11. When the boundary displacement $U_{a}$ approaches the measured catastrophic rupture $U_{F}$, the power law presents well, and the predictions of catastrophic rupture are also good, as $U_{F}^{P}$ tends to 1 as shown in Fig. $11 \mathrm{~b}$. Therefore, when the boundary of the available data $U_{a}$ approaches $U_{F}$, the prediction improves. It can be seen that the

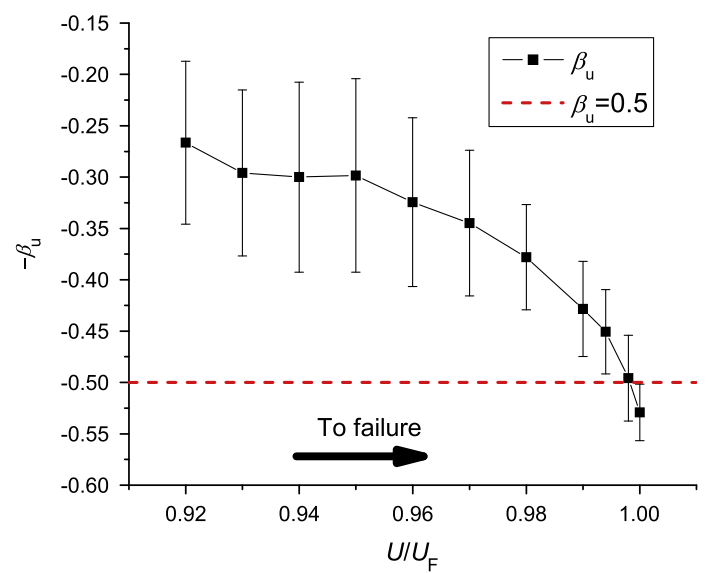

Fig. 6. The average value of $\beta_{u}$ and the corresponding error bars for five specimens.
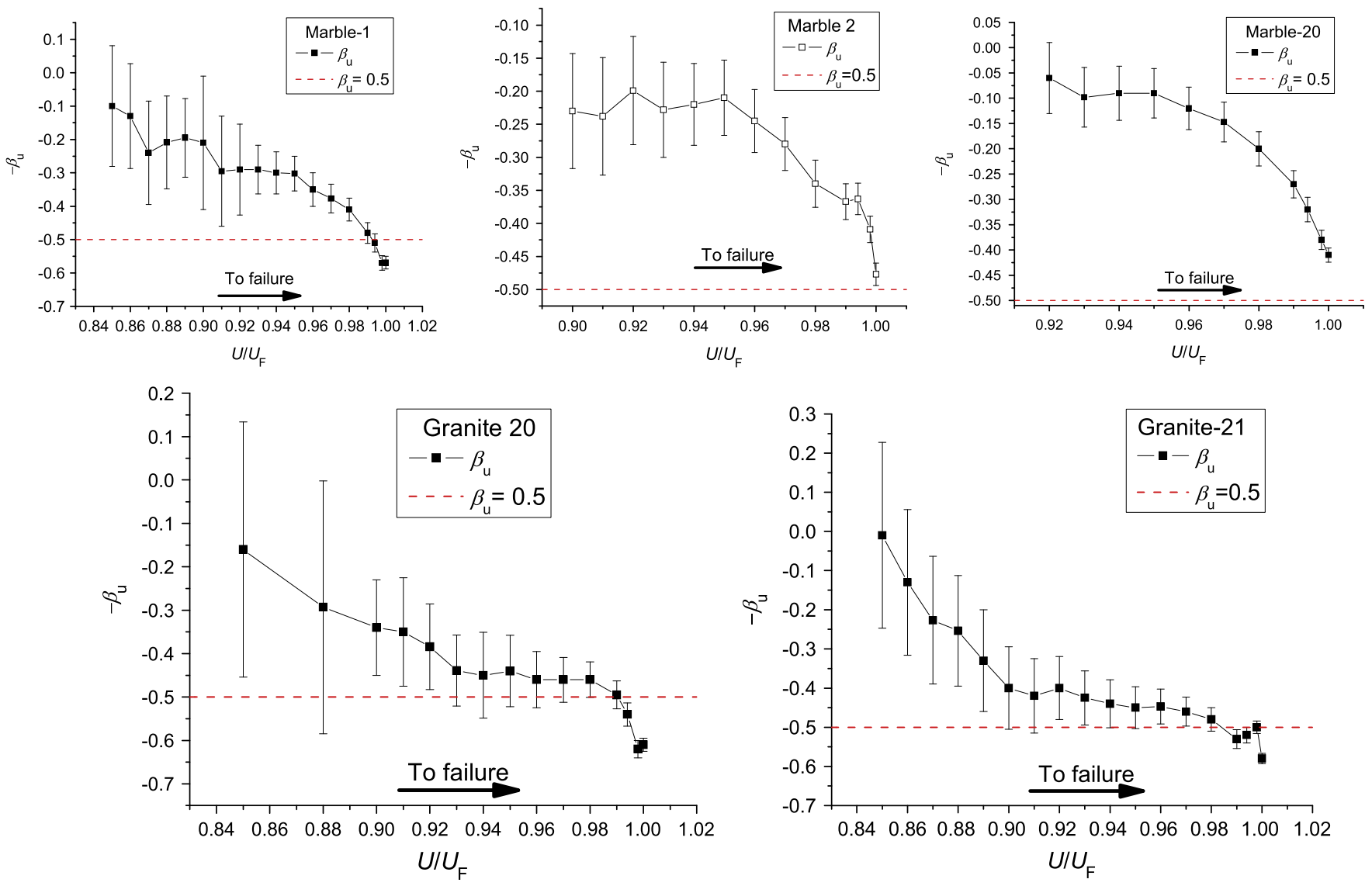

Fig. 5. The evolving behaviors of $\beta_{u}$ with corresponding error bars for five samples. 

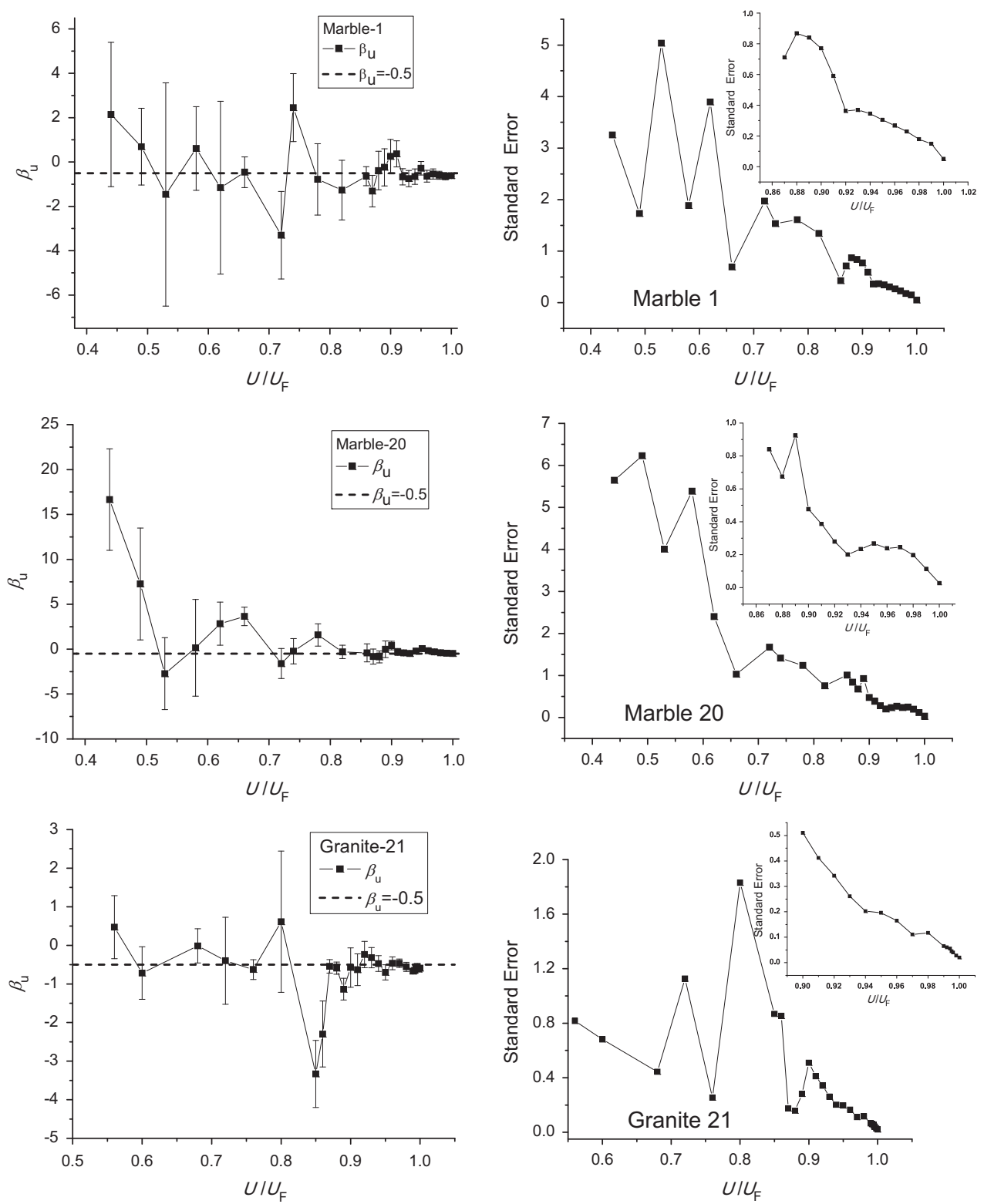

Fig. 7. The fitted results of $\beta_{u}$ and the standard errors obtained by a moving window with a fixed window size of $4 \%$ of $U_{F}$ (three specimens).

experimental implies that the present approach may work well for short-term forewarning of catastrophic rupture. For practical applications, a more effective algorithm is still needed.

\section{Discussions and conclusions}

(1) Catastrophic rupture and its physical essence. Catastrophic rupture can be described as the critical state, where an infinitesimal increment of the external controlling variable will result in a finite increment of the accumulation but an infinite increase in its response. In essence, the evolution of the macroscopic response depends on the micro-physical properties and microdamage process of the sample, which induces the sample-specificity of catastrophic rupture [50,51]. Generally, no testing machine is completely stiff. Moreover, in testing heterogeneous materials, the occurrence of catastrophic rupture will depend not only on the stiffness of the loading machine but also on the damage evolution and elastic unloading in the heterogeneous specimen. The greater the elastic unloading energy release coming from both the elastic machine and the elastic unloading part of the sample, the more prone the sample is to catastrophic rupture, and vice versa. The catastrophic rupture (very localized) in granite and gradual rupture (very diffuse damage) in concrete in our tests are two typical cases.

The cross-check of experimental observations in rocks demonstrates that the observed power-law singularity near catastrophic rupture should be attributed to the interaction between the heterogeneities at meso-scales. This deepens the previous physical understanding of the transition from accumulation and coalescence of microcracks and microdefects to catastrophic rupture $[11,40]$.

In spite of the complicated features of catastrophic rupture, the experimental results demonstrate a common critical power-law singularity of the macroscopic response ahead of catastrophic rupture.

(2) Singular response but finite cumulative quantity. When approaching catastrophic rupture, the singular power law appears in the response, while the corresponding cumulative 
Table 1

The measured Values of $\beta_{u}$ in rock experiments.

\begin{tabular}{|c|c|c|c|c|c|}
\hline Material of rock & No. of specimen & $\begin{array}{l}\text { Fitted values of } \beta_{u} \\
\text { and its standard error }\end{array}$ & Material of rock & No. of specimen & $\begin{array}{l}\text { Fitted values of } \beta_{u} \\
\text { and its standard error }\end{array}$ \\
\hline \multirow[t]{23}{*}{ Granite } & $\mathrm{G}-1$ & $0.53 \pm 0.05$ & \multirow[t]{23}{*}{ Marble } & M-1 & $0.61 \pm 0.05$ \\
\hline & $\mathrm{G}-2$ & $0.43 \pm 0.04$ & & M-2 & $0.63 \pm 0.04$ \\
\hline & $G-3$ & $0.46 \pm 0.05$ & & M-3 & $0.52 \pm 0.03$ \\
\hline & $\mathrm{G}-4$ & $0.50 \pm 0.04$ & & $\mathrm{M}-4$ & $0.38 \pm 0.03$ \\
\hline & G-5 & $0.37 \pm 0.06$ & & M-5 & $0.47 \pm 0.03$ \\
\hline & G-6 & $0.40 \pm 0.06$ & & M-6 & $0.45 \pm 0.04$ \\
\hline & G-7 & $0.41 \pm 0.06$ & & M-7 & $0.52 \pm 0.05$ \\
\hline & $\mathrm{G}-8$ & $0.43 \pm 0.05$ & & M-8 & $0.52 \pm 0.03$ \\
\hline & G-9 & $0.71 \pm 0.12$ & & M-9 & $0.69 \pm 0.05$ \\
\hline & G-10 & $0.53 \pm 0.05$ & & M-10 & $0.72 \pm 0.06$ \\
\hline & G-11 & $0.45 \pm 0.05$ & & $\mathrm{M}-11$ & $0.45 \pm 0.03$ \\
\hline & G-12 & $0.64 \pm 0.08$ & & $\mathrm{M}-12$ & $0.42 \pm 0.02$ \\
\hline & G-13 & $0.55 \pm 0.09$ & & M-13 & $0.46 \pm 0.02$ \\
\hline & G-14 & $0.62 \pm 0.06$ & & $\mathrm{M}-14$ & $0.50 \pm 0.03$ \\
\hline & G-15 & $0.72 \pm 0.05$ & & M-15 & $0.44 \pm 0.03$ \\
\hline & G-16 & $0.41 \pm 0.04$ & & M-16 & $0.46 \pm 0.03$ \\
\hline & G-17 & $0.59 \pm 0.03$ & & $\mathrm{M}-17$ & $0.50 \pm 0.03$ \\
\hline & G-18 & $0.38 \pm 0.02$ & & M-18 & $0.42 \pm 0.03$ \\
\hline & G-19 & $0.62 \pm 0.03$ & & M-19 & $0.54 \pm 0.03$ \\
\hline & G-20 & $0.65 \pm 0.03$ & & $\mathrm{M}-20$ & $0.48 \pm 0.03$ \\
\hline & G-21 & $0.59 \pm 0.02$ & & & \\
\hline & G-22 & $0.50 \pm 0.03$ & & & \\
\hline & G-23 & $0.49 \pm 0.02$ & & & \\
\hline Mean value of $\beta_{u}$ & and its standard e & or: $\mathbf{0 . 5 1} \pm \mathbf{0 . 1 0}$ & & & \\
\hline
\end{tabular}

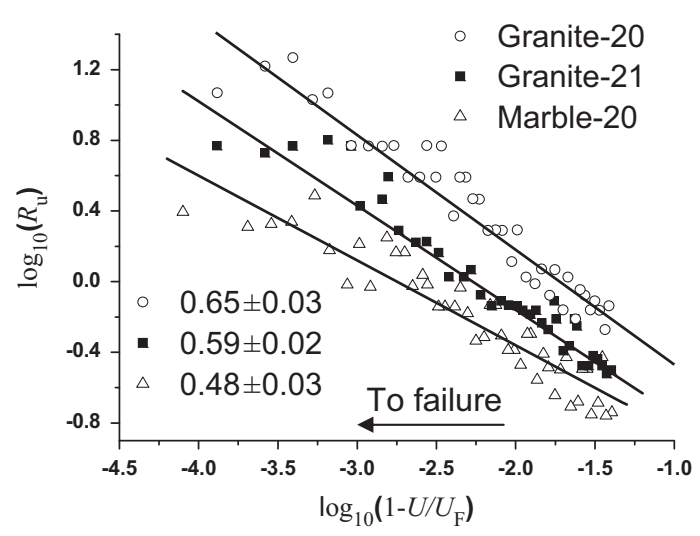

Fig. 8. The log-log data (symbols) and fittings (solid lines) of the response $R_{u}=\mathrm{d} u / \mathrm{d} U$ versus the reduced displacement for three samples.

quantity should remain finite. As noted by Ben-Zion and Lyakhovsky [15], any power law leading to an unrealistic singular cumulative energy would be physically unreasonable [38].

(3) Governing variable. In general, provided a proper governing variable is identified, a power-law singularity of responses appearing in advance of catastrophic rupture can be unveiled. However, for forecasting practice, it is more helpful to take the time $t$ as the independent variable, as in the well-known power-law time-to-rupture of cumulative Benioff strain release $\left(1-t / t_{F}\right)^{m}$. Therefore, a transformation from the governing variable into time $t$ is needed in practice. However, it should be pointed out that, for the time being, the present observed power-law singularity (about -0.5) cannot be simply compared to the power laws for the time to rupture in earthquakes, as $\left(t_{f}-t\right)^{m}$, with $m$ close to 0.3 . Apart from the huge differences between crust and rock samples in the laboratory, the reasons are at least two-fold. First, the independent variable used in the power law $\left(t_{f}-t\right)^{m}$ is time $t$, whereas the observed power law singularity $(-0.5)$ must be referred to a controlling variable, such as the cross-head displacement $U$ in laboratory tests. Second, the dependent variable in earthquakes is the Benioff strain, whereas in our laboratory tests it is the deformation of the sample. The interval of acceleration has been shown to decrease systematically with respect to decreasing strain rate [52], and thus the proportion of time for rapidly accelerating nucleation may be much smaller in the Earth. Amoruso and Crescentini [53] reported that even seconds before the 2009 L'Aquila earthquake in Italy, "strain is stable at the 10-12 level and pre-rupture nucleation slip in the hypocentral region is constrained to have a moment less than $2 \times 1012 \mathrm{Nm}$, i.e. $0.00005 \%$ of the main shock seismic moment". So the nucleation processes observed here may be restricted in the Earth to a very small patch at the nucleation depth ( $\sim 10 \mathrm{~km}$ in this case) that in practice may be undetectable. Thus, to correlate the observed power singularity in the laboratory to real earthquakes, much more work is badly needed, for instance, to explore the similarities between experimental tests and seismic cycles.

(4) Common power-law singularity but sample-specific threshold. The reported power-law singularity $A\left(1-U / U_{F}\right)^{-\beta}$ includes two opposite but interrelated features of catastrophic rupture: the power-law singularity always appears ahead of catastrophic rupture but the threshold $U_{F}$ shows obvious sample-specificity. The reason for this is that the transition from gradual accumulation of damage to catastrophic rupture follows the same mode (second-order asymptotic approximation appears to be valid in the vicinity of catastrophic rupture) but the transition itself (the threshold) strongly depends on the critical coalescence of damage events in a specific sample.

In our experiments, the scatter of the responses appearing in the vicinity of rupture may result mainly from two facts. The first is that the testing machine cannot control loading well when approaching catastrophic rupture, i.e., the governing displacement $U$ cannot be a smooth variable in practice, even if the loading is quasi-static. The second is more inherent: the powerlaw singularity usually describes the characteristics of quasicontinuous evolution, however, the evolution appears as a time-series of small (much smaller than the catastrophic event) 
a

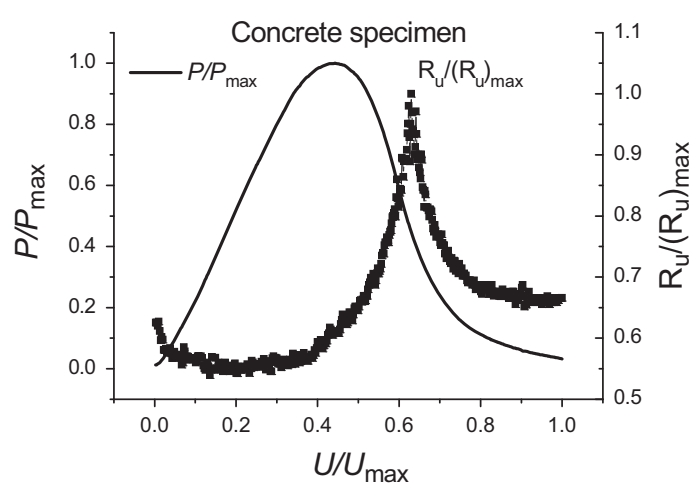

b

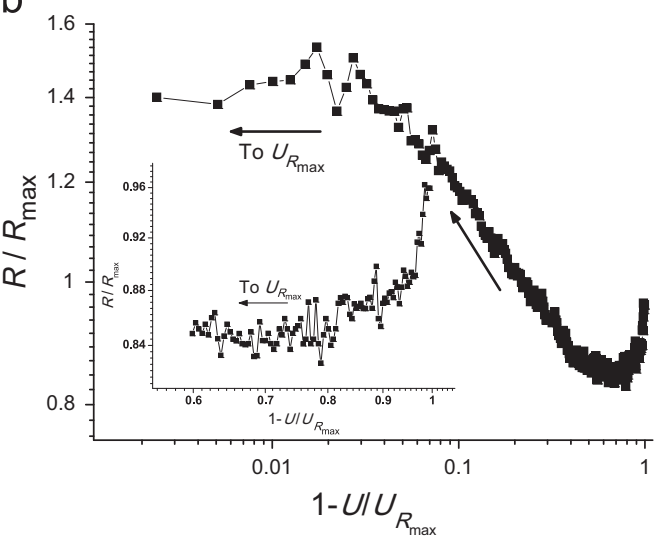

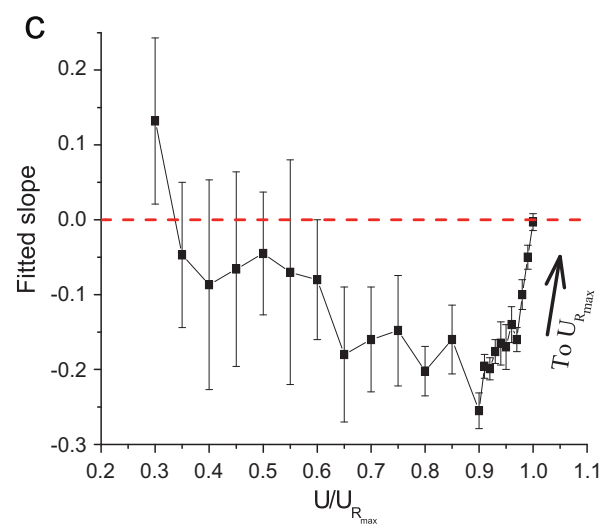

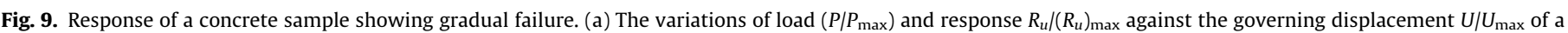

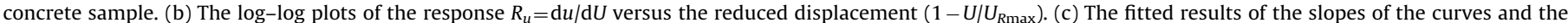
standard errors.
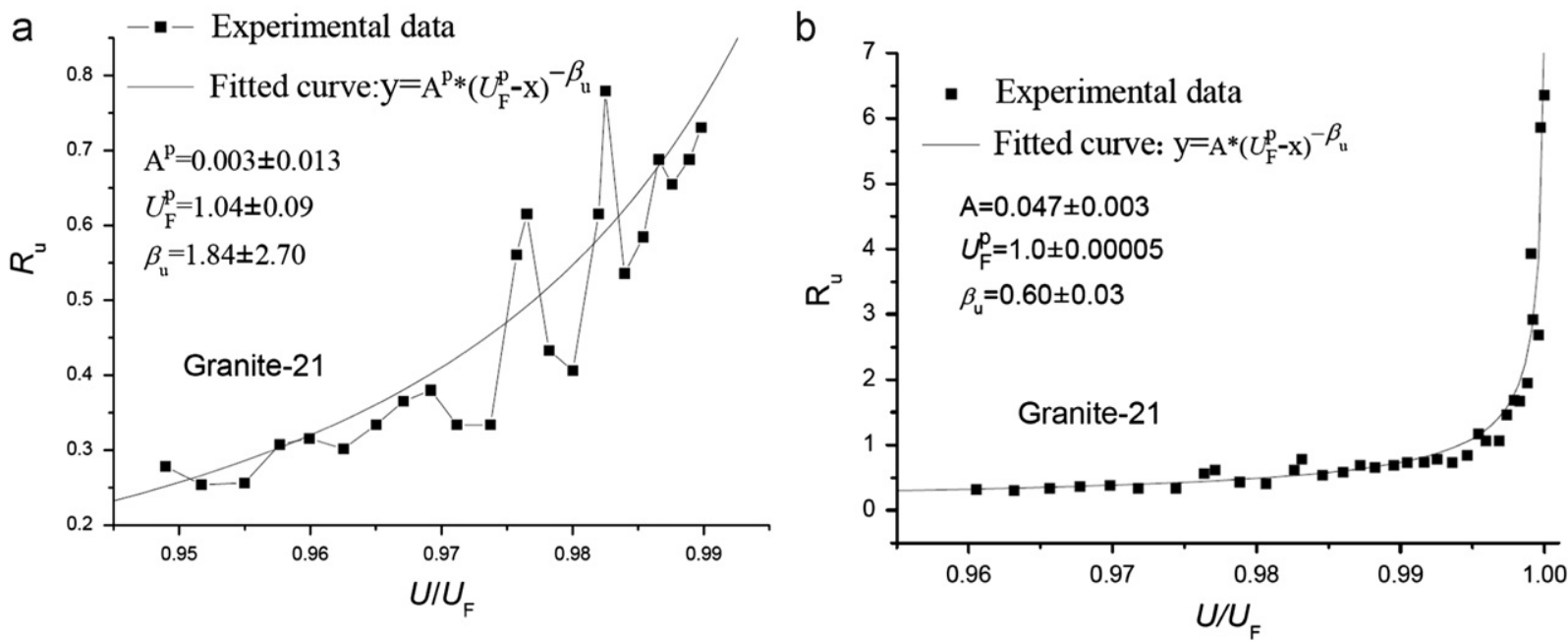

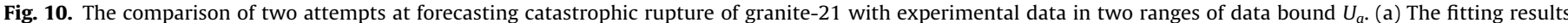
with $U_{a}=0.99 U_{F}$ and the data ranging from $96 \%$ of $U_{a}$ to $U_{a}$. (b) The fitting result based on the data ranging from $96 \%$ of $U_{F}$ to $U_{F}$.

discrete and intermittent events. Additionally, the value $A$ in response is sensitively dependent on a number of variables such as loading modes, the stiffness ratio, localized damage and the details of damage pattern.

(5) Loading modes. It is also important to note that Guarino et al. $[13,49]$ reported a power-law increase in the energy associated with acoustic emissions prior to rupture in fiberboard panels under rapid loading, and Turcotte and Shcherbakov [11] presented a discussion of the temporal scaling laws regarding time delay based on specifying the kinetic equation for damage evolution. However, the reported power-law singularity in this paper is obtained when the rock specimens are subjected to 
a

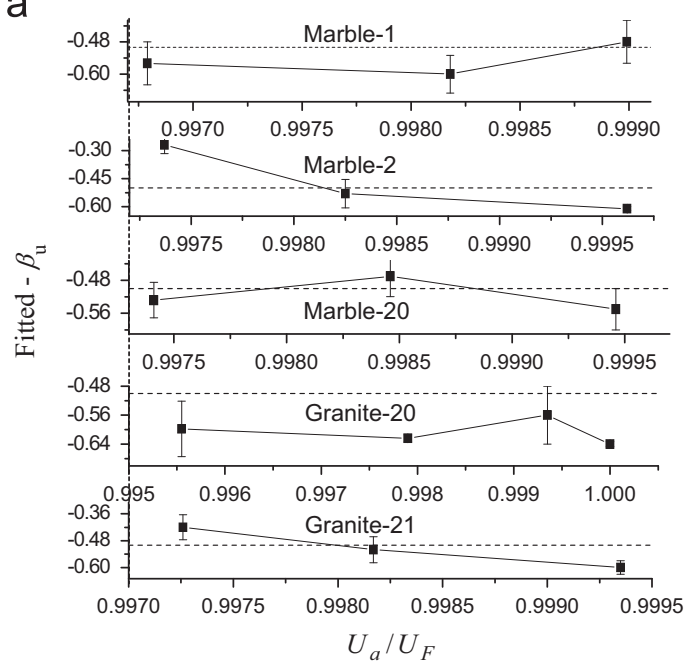

b

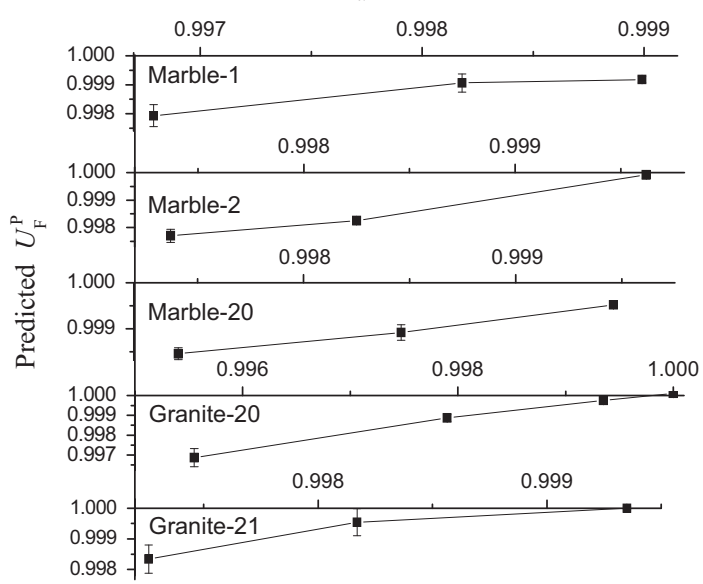

Fig. 11. The predictive fittings with various data bound $U_{a}$ before catastrophic rupture $U_{F}$ (five samples). (a) Fitted values of exponent $-\beta_{\mathrm{u}}$ with their error bars. The dashed line indicates $-\beta_{\mathrm{u}}=-0.5$. (b) The predicted catastrophe point $U_{F}^{P}$ with their error bars at various $U_{a}$ ahead of catastrophe.

monotonically quasi-static loading. Whether the results presented in this paper could be applied to the cases under creep or rapid loading should be further investigated.

In summary, this paper reports a power-law singularity for the responses prior to catastrophic rupture based on rock experiments. Moreover, gradual rupture does not present such a singularity, though its responses can increase before rupture. Therefore, this power-law singularity will have significant potential applications in forecasting failure in rock and concrete under engineering conditions.

\section{Acknowledgements}

This work is supported by the National Natural Science Foundation of China (Grant nos. 90715001, 11021262, 10572139 and 10802073), the National Basic Research Program of China (973 Program 2007CB814800) and the CAS Innovation Program. The computations were performed on the PC clusters of State Key Laboratory of Scientific and Engineering Computing of Chinese Academy of Sciences and Shenteng6800 of Supercomputing Center, Computer Network Information Center, Chinese Academy of Sciences.

\section{References}

[1] Geller RJ, Jackson DD, Kagan YY, Mulargia F. Earthquakes cannot be predicted. Science 1997;275:1616-7.

[2] Wyss M. Cannot earthquakes be predicted? Science 1997;278:487-90.

[3] 〈http://www.nature.com/nature/debates/earthquake/equake_frameset. html $\rangle ; 1999$ [Accessed 25.02.99].

[4] Knopoff L. Earthquake prediction: the scientific challenge. Proc Natl Acad Sci USA 1996;93(9):3719-20.

[5] Rundle JB, Turcotte DL, Shcherbakov R, Klein W, Sammis C. Statistical physics approach to understanding the multiscale dynamics of earthquake fault systems. Rev Geophys 2003;41:1019-48.

[6] Xia MF, Wei YJ, Ke FJ, Bai YL. Critical sensitivity and trans-scale fluctuations in catastrophic failure. Pure Appl Geophys 2002;159:2491-509.

[7] Weatherley D, Mora P. Accelerating precursory activity within a class of earthquake analogue automata. Pure Appl Geophys 2004;161:2005-19.

[8] Dahmen K. Nonlinear dynamics: universal clues in noisy skews. Nat Phys 2005; $1: 13-4$

[9] Hough S. Predicting the unpredictable: the tumultuous science of earthquake prediction. Princeton: Princeton University Press; 2009.

[10] Vere-Jones D. Statistical theories of crack propagation. Math Geol 1977;9(5):455-81.

[11] Turcotte DL, Shcherbakov R. Can damage mechanics explain temporal scaling laws in brittle fracture and seismicity? Pure Appl Geophys 2006;163: 1031-45.

[12] Garcimartín A, Guarino A, Bellon L, Ciliberto S. Statistical properties of fracture precursors. Phys Rev Lett 1997;79:3202-5.

[13] Guarino A, Garcimartin A, Ciliberto S. An experimental test of the critical behaviour of fracture precursors. Eur Phys J B 1998;6:13-24.

[14] Yin XC, Chen XZ, Song ZP, Yin C. A new approach to earthquake prediction: The load/unload response ratio (LURR) theory. Pure Appl Geophys 1995;145: 701-15.

[15] Ben-Zion Y, Lyakhovsky V. Accelerated seismic release and related aspects of seismicty patterns on earthquake faults. Pure Appl Geophys 2002;159: $2385-412$.

[16] Johansen A, Sornette D. Critical ruptures. Eur Phys J B 2000;18:163-81.

[17] Nechad H, Helmstetter A, Guerjouma R, El, Sornette D. Creep ruptures in heterogeneous materials. Phys Rev Lett 2005;94:045501.

[18] Xia MF, Ke FJ, Bai J, Bai YL. Threshold diversity and trans-scales sensitivity in a finite nonlinear evolution model of materials failure. Phys Lett A 1997;236:60-4.

[19] Vere-Jones D, Robinson R, Yang W. Remarks on the accelerated moment release model-problems of model formulation, simulation and estimation. Geophys J Int 2001:144:517-31.

[20] Jaumé SC, Sykes LR. Evolving towards a critical point: A review of accelerating seismic moment/energy release prior to large and great earthquake. Pure Appl Geophys 1999:155:279-306.

[21] Das S, Scholz CH. Theory of time-dependent rupture in the Earth. J Geophys Res 1981;108(B2):6039-51, http://dx.doi.org/10.1029/JB086iB07p06039.

[22] Main I. Prediction of failure times in the earth for a time-varying stress. Geophys J Int 1988;92:455-64.

[23] Rundle JB, Klein W, Turcotte DL, Malamud BD. Precursory seismic activation and critical-point phenomena. Pure Appl Geophys 2000;157:2165-82.

[24] Zapperi SP, Ray HE, Stanley A, Vespignani P. First-order transition in the breakdown of disordered media. Phys Rev Lett 1997;78:1408-11.

[25] Moreno Y, Gómez JB, Pacheco AF. Fracture and second-order phase transitions. Phys Rev Lett 2000;85:2865-8.

[26] Sornette D. Mean-field solution of a block-spring model of earthquakes. J Phys I France 1992;2:2089-96.

[27] Hardebeck JL, Felzer KR, Michael AJ. Improved tests reveal that the accelerating moment release hypothesis is statistically insignificant. J Geophys Res 2008;113:B08310, http://dx.doi.org/10.1029/2007JB005410.

[28] Mignan A, King GCP, Bowman DD, Lacassin R, Dmowska R. Seismic activity in the Sumatra-Java region prior to the December 26, $2004(\mathrm{Mw}=9.0-9.3)$ and March 28, $2005(\mathrm{Mw}=8.7)$ earthquakes. Earth Planet Sci Lett 2006;244: 639-54.

[29] Carlson JM, Doyle J. Highly optimized tolerance:a mechanism for power laws in designed systems. Phys Rev E 1999;60:1412-27.

[30] Dahmen K, Sethna JP. Hysteresis, avalanches, and disorder induced critical scaling: a renormalization group approach. Phys Rev B 1996;53:14872-905.

[31] Acharyya M, Chakrabarti BK. Growth of breakdown susceptibility in random composites and the stick-slip model of earthquakes: prediction of dielectric breakdown and other catastrophes. Phys Rev E 1996;53:140-7.

[32] Rong F, Wang HY, Xia MF, Ke FJ, Bai YL. Catastrophic failure induced damage coalescence in heterogeneous brittle media. Pure Appl Geophys 2006;163: 1847-65.

[33] Vallianatos F, Triantis D, Tzanis A, Anastasiadis C, Stavrakas I. Electric earthquake precursors: from laboratory results to field observations. Phys Chem Earth 2004;29:339-51.

[34] Ohnaka M. A constitutive scaling law and a unified comprehension for frictional slip failure, shear fracture of intact rock, and earthquake rupture. J Geophys Res 2003;108(B2):2080, http://dx.doi.org/10.1029/2000JB000123.

[35] Ohnaka M. Rupture in the laboratory. Science 2004;303(5665):1788-9.

[36] Xia K, Rosakis AJ, Kanamori H. Laboratory earthquakes: the sub-Rayleigh-tosupershear rupture transition. Science 2004;303(5665):1859-61. 
[37] Lu X, Rosakis AJ, Lapusta N. Rupture modes in laboratory earthquakes: effect of fault prestress and nucleation conditions. J Geophys Res 2010;115:B12302.

[38] Main IG. A damage mechanics model for power-law creep and earthquake aftershock and foreshock sequences. Geophys J Int 2000;142:151-61.

[39] Scholz CH. The frequency-magnitude relation of microfracturing in rock and its relation to earthquakes. Bull Seismol Soc Am 1968;58(1):399-415.

[40] Lockner D. The role of acoustic-emission in the study of rock fracture. Int J Rock Mech Min Sci 1993;30:883-99.

[41] Greenhough J, Bell AF, Main IG. Comment on "Relationship between accelerating seismicity and quiescence, two precursors to large earthquakes" by Arnaud Mignan and Rita Di Giovambattista. Geophys Res Lett 2009 L17303.

[42] Wawersik WR, Fairhurst C. A study of brittle rock fracture in laboratory compression. Int J Rock Mech Min Sci 1970;7(5):561-75.

[43] Labuz JF, Biolzi L. Class I vs Class II stability: a demonstration of size effect. Int J Rock Mech Min Sci 1991;28(2/3):199-205.

[44] Hao SW, Wang HY, Xia MF, Ke FJ, Bai YL. Relationship between strain localization and catastrophic failure. Theor Appl Fract Mech 2007;48:41-9.

[45] Hao SW, Xia MF, Ke FJ, Bai YL. Evolution of localized damage zone in heterogeneous media. Int J Damage Mech 2010;19:787-804.
[46] Jaeger JC, Cook NGW, Zimmerman RW. Fundamentals of rock mechanics. 4th ed. Oxford: Wiley-Blackwell; 2007.

[47] Rudnicki JW, Rice JR. Conditions for the localization of deformation in pressure-sensitive dilatant materials. J Mech Phys Solids 1975;23:371-94.

[48] Bell AF, Greenhough J, Heap MJ, Main IG. Challenges for forecasting based on accelerating rates of earthquakes at volcanoes and laboratory analogues. Geophys J Int 2011;185:718-23.

[49] Guarino A, Ciliberto S, Garcimartin A, Zei M, Scorretti R. Failure time and critical behaviour of fracture precursors in heterogeneous materials. Eur Phys J B 2002;26:141-51.

[50] Xia MF, Song ZQ, Xu JB, Zhao KH, Bai YL. Sample specific behaviour in failure models of disordered media. Commun Theor Phys 1996;25:49-54.

[51] Bai YL, Wang HY, Xia MF, Ke FJ. Statistical mesomechanics of solid, liking coupled multiple space and time scales. Appl Mech Rev 2005;58:372-88.

[52] Ojala IO, Main IG, Ngwenya BT. Strain rate and temperature dependence of Omori law scaling constants of $\mathrm{AE}$ data: implications for earthquake foreshock-aftershock sequences. Geophys Res Lett 2004;31:L24617.

[53] Amoruso A, Crescentini L. Limits on earthquake nucleation and other preseismic phenomena from continuous strain in the near field of the 2009 L'Aquila earthquake. Geophys Res Lett 2010;37:L10307. 